\title{
Severe, Refractory Immune Thrombocytopenia Occurring After SARS-CoV-2 Vaccine
}

This article was published in the following Dove Press journal:

Journal of Blood Medicine

\author{
Jackie M Helms $\mathbb{D}^{\prime}$ \\ Kristin T Ansteatt $\mathbb{D}^{1}$ \\ Jonathan C Roberts (D) ${ }^{1,2}$ \\ Sravani Kamatam ${ }^{3}$ \\ Kap Sum Foong $\mathbb{( D}^{2,3}$ \\ Jo-mel S Labayog ${ }^{4}$ \\ Michael D Tarantino ${ }^{1,2}$ \\ 'The Bleeding and Clotting Disorders \\ Institute, Peoria, IL, USA; ${ }^{2}$ Department of \\ Medicine, University of Illinois College of \\ Medicine-Peoria, Peoria, IL, USA; \\ ${ }^{3}$ Department of Medicine, Saint Francis \\ Medical Center, Peoria, IL, USA; \\ ${ }^{4}$ Department of Medicine, OSF Sacred \\ Heart Medical Center, Danville, IL, USA
}

Correspondence: Michael D Tarantino Chief Medical Officer, The Bleeding and Clotting Disorders Institute, 9128 North Lindbergh Drive, Peoria, IL, 61615, USA

Tel + I 309 692-5337

Fax +I 309 693-3913

Email mtarantino@ilbcdi.org
Abstract: The rollout of the SARS-CoV-2 vaccine is underway, and millions have already been vaccinated. At least 25 reports of "immune thrombocytopenia" (ITP) or "thrombocytopenia" following the Moderna or Pfizer vaccine have been added to the Vaccine Adverse Event Reporting System (VAERS) in the US. ITP is a rare but known complication of several vaccinations. SARS-CoV-2 vaccine is new, with a novel mechanism of action, and understanding the epidemiology, clinical manifestations, treatment success and natural history of post-vaccination thrombocytopenia is evolving. We report a 74-year-old man who developed refractory thrombocytopenia within one day of receiving the Moderna SARS-CoV-2 vaccine. Several hours after vaccination, he developed significant epistaxis and cutaneous purpura. Severe thrombocytopenia was documented the following day, and he developed extremity weakness and encephalopathy with facial muscle weakness. Over a 14-day period, thrombocytopenia was treated first with high dose dexamethasone, intravenous immunoglobulin, platelet transfusions, rituximab, plasma exchange (for presumed acute inflammatory demyelinating polyneuropathy (AIDP)), and four daily doses of the thrombopoietin receptor agonist (TPO-RA) eltrombopag (Promacta ${ }^{\mathrm{TM}}$ ), without a platelet response. Three days later, he received the TPO-RA romiplostim (Nplate ${ }^{\mathrm{TM}}$ ). Five days later, his platelet count began to rise and by post-vaccination day 25 , his platelet count was in the normal range. Thrombocytopenia was refractory to frontline and second-line treatment. The eventual rise in his platelet count suggests that one or both TPO-RAs may have impacted platelet recovery. Possibly, but less likely given the temporality, the drug-induced thrombocytopenia was subsiding. The aggressive use of immunosuppressive treatment may jeopardize the intended purpose of the SARS-CoV-2 vaccine, and earlier use of non-immunosuppressive second-line treatment for vaccine-related severe thrombocytopenia, such as with TPO-RAs, should be considered. While it is imperative to continue the global vaccination program, vigilance to the occurrence of post-vaccination severe thrombocytopenia is warranted.

Keywords: immune thrombocytopenic purpura, platelet, SARS-CoV-2, thrombocytopenia, thrombopoietin receptor agonist, vaccine

\section{Introduction}

Primary Immune Thrombocytopenic Purpura (ITP) is an autoimmune disorder characterized by increased platelet destruction and decreased platelet production. ${ }^{1}$ The incidence of ITP is 6 per 100,000 adults/year. ${ }^{1}$ ITP has been documented as a result of viral illnesses or vaccinations that are given for prevention of infectious illnesses. $^{2-11}$ The recent global pandemic of SARS-CoV-2, a novel coronavirus, has caused many deaths worldwide and a global vaccination program is imperative to achieving herd immunity. ${ }^{12,13}$ Platelet aggregation and activation appear to be 
deranged in hospitalized patients with COVID-19, however the effect on the platelet count is not significantly correlated, although severe (presumed immune-mediated) thrombocytopenia during COVID-19 has been reported in a small number of patients. ${ }^{14-17}$ To date, the SARS-CoV-2 vaccine appears to rarely cause an immune-mediated platelet destruction, not unlike that seen with the rubella, pneumococcus, and influenza vaccines. ${ }^{2,4,5}$ The mechanism of post-vaccination thrombocytopenia, in both live and inactivated vaccines, is presumed to be immune mediated and may be similarly related to hyperfunction of B-cells observed in ITP. ${ }^{18,19}$ Tens of millions of people have been vaccinated with the new SARS-CoV-2 vaccine. A small but growing number of cases of "immune thrombocytopenia" or "thrombocytopenia" following the administration of the SARS-CoV-2 vaccine have been reported to the FDA's VAERS. ${ }^{20}$ Review of available information regarding post SARS-CoV-2 vaccination-related thrombocytopenia suggests a heterogenous onset, severity, and duration. At least one reported patient had a good platelet response to frontline treatment with corticosteroids and IVIg. ${ }^{21}$ Here we report a case of severe, multi-drug, refractory immune thrombocytopenia shortly after the initial dose of the Moderna SARS-CoV-2 vaccine. The thrombocytopenia eventually relented after treatment with the thrombopoietin receptor agonist (TPO-RA) romiplostim.

\section{Case Report}

A 74-year-old male with hypertension, gout, hyperlipidemia and nonischemic cardiomyopathy, presented with acute epistaxis and diffuse cutaneous purpura a few hours after receiving the first dose of the Moderna SARS-CoV2 vaccine. He received the influenza and pneumococcal vaccines three months and six weeks, respectively, prior to his presentation.

Two months prior to vaccination, the patient had a platelet count of $224 \times 10^{9} / \mathrm{L}$. The SARS-CoV-2 vaccine was administered on 19 Jan 2021 and that evening he experienced severe epistaxis and diffuse purpura. The next day, he presented to a local emergency department with uncontrolled epistaxis, and was noted to have a platelet count of 10 x $10^{9} / \mathrm{L}$. He was hospitalized for five days and received highdose dexamethasone ( $40 \mathrm{mg} /$ day), five daily doses of intravenous immunoglobulin $(400 \mathrm{mg} / \mathrm{kg} /$ day $)$, three daily platelet transfusions, and two weekly doses of rituximab $\left(375 \mathrm{mg} / \mathrm{m}^{2} /\right.$ dose). Immune suppression with dexamethasone ( $20 \mathrm{mg} /$ day) was continued after hospital discharge. (See Figure 1) Due to persistent severe thrombocytopenia, platelet count $21 \times 10^{9} / \mathrm{L}$, the TPO-RA eltrombopag $(50 \mathrm{mg} /$ day $)$ was initiated on postvaccination day ten.

On post-vaccination day 13 , he again presented to the emergency department with severe thrombocytopenia, platelet count $12 \times 10^{9} / \mathrm{L}$, but now with progressive, generalized weakness that first involved the left arm and the lower

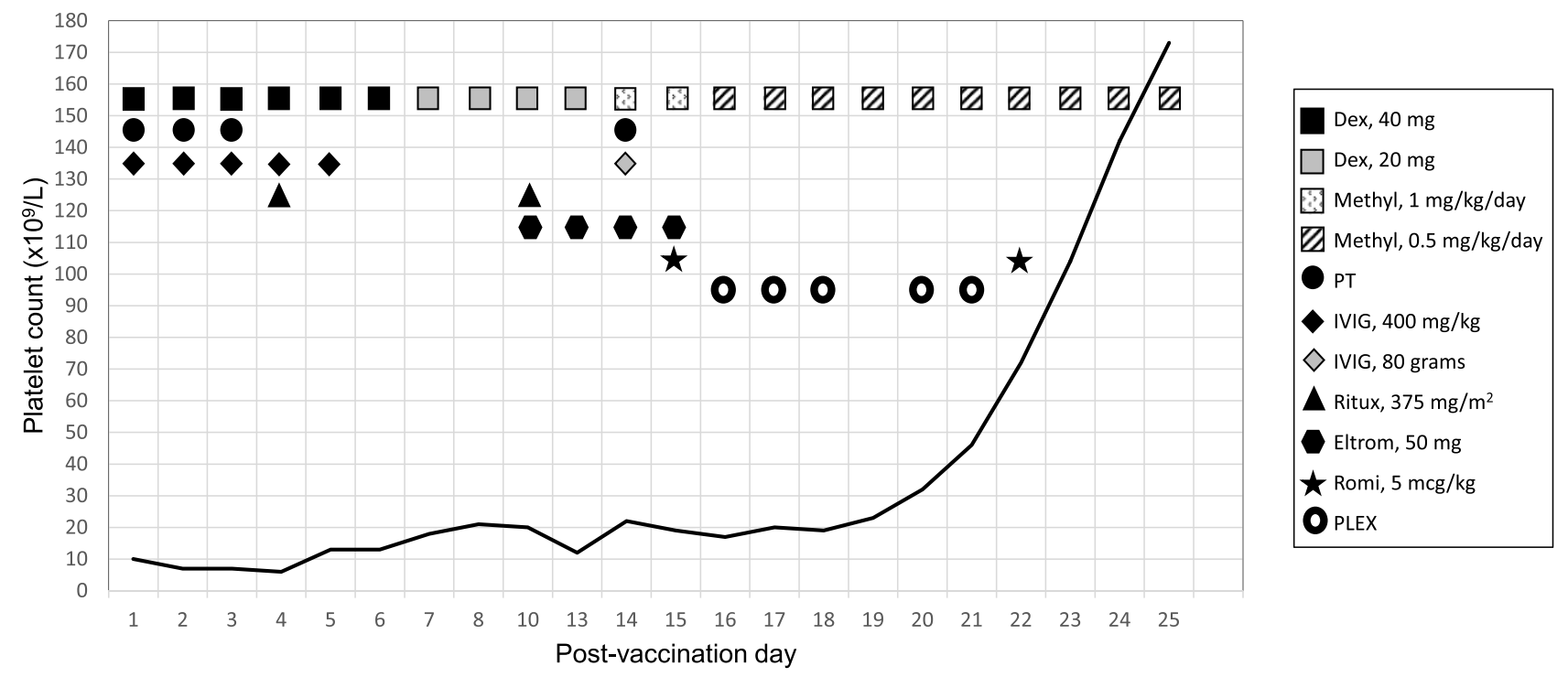

Figure I Post-vaccination platelet count.

Abbreviations: Dex, dexamethasone; Methyl, methylprednisolone; PT, platelet transfusion; IVIG, intravenous immunoglobulin; Ritux, rituximab; Eltrom, eltrombopag; Romi, romiplostim; PLEX, plasma exchange. 
extremities bilaterally, back pain causing inability to ambulate, urinary retention, constipation and encephalopathy with dysarthria. These findings, although concurrent with the persistent, refractory severe thrombocytopenia, were thought to be unrelated. Due to suspicion of acute inflammatory demyelinating polyneuropathy (AIDP), plasma exchange was initiated on post-vaccination day 15. Magnetic resonance imaging on post-vaccination day 16 revealed severe L1-5 stenosis with multi-level disc herniation and fluid collections within the lumbar, posterior and paraspinal musculature.

On post-vaccination day 14 , he received one pheresis unit of platelets for a platelet count of $22 \times 10^{9} / \mathrm{L}$ without improvement in his platelet count 1-hour post transfusion, and an additional dose of IVIg of 80 grams was given. On post-vaccination day 15 , he received high dose methylprednisolone $(1 \mathrm{mg} / \mathrm{kg} /$ day $)$ and romiplostim $(5 \mathrm{mcg} / \mathrm{kg})$ with no increase in the platelet count for the next five days (See Figure 1).

Examination of the peripheral blood smear on postvaccination day 14 revealed normal-to-large sized platelets. Blood and urine cultures grew methicillin-susceptible Staphylococcus aureus (MSSA) on post-vaccination day 13 that was treated with cefazolin. SARS-CoV-2 by a polymerase chain reaction assay was not detected on post-vaccination days 13 and 14. Additionally, tests for human immunodeficiency virus, Hepatitis B virus (HBV), Hepatitis C virus, Epstein-Barr virus, cytomegalovirus, and parvovirus B19 were negative.

On post-vaccination day 19, after the third episode of plasma exchange, his facial weakness improved. On postvaccination day 22 , his platelet count was $72 \times 10^{9} / \mathrm{L}$, a second dose of romiplostim $5 \mathrm{mcg} / \mathrm{kg}$ was administered, and a corticosteroid taper was begun. He was transferred to a skilled nursing facility on post-vaccination day 25 with a platelet count of $173 \times 10^{9} / \mathrm{L}$.

\section{Discussion}

Previous studies have shown that ITP is a rare complication following routine vaccinations, primarily rubella but also pneumococcus, Haemophilus influenza type B, HBV, human papilloma virus, varicella-zoster, diphtheria, tetanus, pertussis, and polio. ${ }^{2-11}$

Our patient presented with severe thrombocytopenia within one day of receiving the Moderna SARS-CoV-2 vaccine with virtually no response to standard ITP treatment, suggesting refractoriness or a different pathophysiology for the severe thrombocytopenia. The patient's refractory thrombocytopenia ultimately responded after treatment with romiplostim (Nplate), much like many cases of ITP with an onset of action as soon as four days after the subcutaneous first dose. ${ }^{22,23}$ This patient's poor response to frontline and second-line treatments suggests that TPO-RA agents may be useful in refractory ITP post SARS-CoV-2 vaccination.

Because of the concurrent, suspected AIDP and concern for jeopardizing the effect of the SARS-CoV-2 vaccine, thereafter, nonimmune suppressive treatment for severe thrombocytopenia was chosen. The deleterious effects of aggressive immunosuppression for thrombocytopenia should not be overlooked. In prior studies, immunosuppressive therapies post vaccination resulted in attenuation of the immune response to the administered vaccine. $^{24}$ TPO-RAs are not immunosuppressive and very effective for persistent or chronic ITP. ${ }^{25}$ Knowing that TPO-RAs may increase the risk of venous or arterial thrombosis in a small proportion of patients, and that patients with SARS-CoV-2 infection are at higher risk for thrombotic events, patients selected for TPO-RA therapy for SARS-CoV-2 related severe thrombocytopenia should be chosen judiciously. ${ }^{26,27}$

\section{Conclusion}

Given the SARS-CoV-2 vaccine is new, with a novel mechanism of action, there exists the uncertainty of whether or not refractory ITP will be a rare adverse event. Notwithstanding that post-vaccination induced ITP is a rare adverse event, it should not limit the use of vaccines, including the SARS-CoV-2 vaccine. However, further investigation is imperative to explicate the pathological mechanism, epidemiology, clinical manifestations, and treatment outcomes.

\section{Consent}

Written informed consent for publication of their details was obtained from the patient. The governing institutional review board (IRB) for St. Francis Medical Center, the University of Illinois College of Medicine- Peoria IRB, gave approval for this case report.

\section{Acknowledgments}

All of the authors wish to acknowledge the diligent work of the clinical and laboratory staffs of OSF Sacred Heart of Mary and OSF Saint Francis Medical Center. 


\section{Disclosure}

Dr Michael D Tarantino reports personal fees for consulting and/or speaking from and past clinical trials investigator for Amgen and Dova, outside the submitted work. The authors report no other conflicts of interest in this work.

\section{References}

1. Weycker D, Hanau A, Hatfield M, et al. Immune thrombocytopenia in US clinical practice: incidence and healthcare burden in the first 12 months following diagnosis. J Med Econ. 2020;23(2):184-192. doi:10.1080/13696998.2019.1669329

2. Black C, Kaye JA, Jick H. MMR vaccine and idiopathic thrombocytopaenic purpura. Br J Clin Pharmacol. 2003;55(1):107-111. doi:10.1046/j.1365-2125.2003.01790.x

3. Garbe E, Andersohn F, Bronder E, et al. Drug induced immune thrombocytopaenia: results from the Berlin Case-Control Surveillance Study. Eur J Clin Pharmacol. 2012;68:821-832. doi:10.1007/s00228-011-1184-3

4. Tseng HF, Sy LS, Quan L. Pneumococcal conjugate vaccine safety in elderly adults. Open Forum Infect Dis. 2018;5(6):1-8.

5. Nagasaki J, Manabe M, Ido K, et al. Postinfluenza vaccination idiopathic thrombocytopenic purpura in three elderly patients. Case Reports in Hematol. Epub 2016 February 21.

6. Jin C, Dong H, Sun Z, et al. Acute immune thrombocytopenic purpura as adverse reaction to oral polio vaccine (OPV). Hum Vaccin Immunother. 2013;9(8):1739-1740. doi:10.4161/hv.24847

7. Genovese C, La Fauci V, Squeri A, Trimarchi G, Squeri R. HPV vaccine and autoimmune diseases: systematic review and metaanalysis of the literature. J Prev Med Hyg. 2018;59:E194-E199. doi:10.15167/2421-4248/jpmh2018.59.3.998

8. Miller E, Waight P, Farrington P, et al. Idiopathic thrombocytopenic purpura and MMR vaccine. Arch Dis Child. 2001;84:227-229. doi:10.1136/adc.84.3.227

9. Cecinati V, Principi N, Brescia L. Vaccine administration and the development of immune thrombocytopenic purpura in children. Human Vacc Immunotherap. 2013;9(5):1158-1162. doi:10.4161/ hv. 23601

10. France EK, Glanz J, Xu S, et al.; Vaccine Safety Datalink Team. Risk of immune thrombocytopenic purpura after measles-mumps-rubella immunization in children. Pediatrics. 2008;121(3):e687-e92. doi:10.1542/peds.2007-1578

11. Isai A, Durand J, Le Meur S, Hidalgo-Simon A, Kurz X. Autoimmune disorders after immunisation with Influenza A/H1N1 vaccines with and without adjuvant: eudraVigilance data and literature review. Vaccine. 2012;30(49):7123-7129. doi:10.1016/j. vaccine.2012.09.032

12. Centers for disease control and prevention.. Available from: https:// www.cdc.gov/coronavirus/2019-ncov/index.html. Accessed February. 15,2021

Journal of Blood Medicine

\section{Publish your work in this journal}

The Journal of Blood Medicine is an international, peer-reviewed, open access, online journal publishing laboratory, experimental and clinical aspects of all aspect pertaining to blood based medicine including but not limited to: Transfusion Medicine; Blood collection, Donor issues, Transmittable diseases, and Blood banking logistics; Immunohematology; Artificial and alternative blood based
13. Omer SB, Yildirim I, Forman HP. Herd Immunity and Implications for SARS-CoV-2 Control. JAMA. 2020;324(20):2095-2096. doi:10.1001/jama.2020.20892

14. Leopold V, Pereverzeva L, Schuurman AR, et al. Platelets are hyperactivated but show reduced glycoprotein VI reactivity in COVID-19 patients. Thromb Haemost. 2021. doi:10.1055/a-1347-5555

15. Comer SP, Cullivan S, Sklanna PB, et al. COVID-19 induces a hyperactive phenotype in circulating platelets. PLoS Biol. 2021;19 (2):e3001109. doi:10.1371/journal.pbio.3001109

16. Slomka A, Kowalewski M, Zekanowska E. Coronavirus disease 2019 (COVID-19): a short review on hematological manifestations. Pathogens. 2020;9(6):493. doi:10.3390/pathogens9060493

17. Bhattacharjee S, Banerjee M. Immune thrombocytopenia secondary to COVID-19: a systematic review. SN Comp Clin Med. 2020;2:2048-2058. doi:10.1007/s42399-020-00521-8

18. Giordano P, Cascioli S, Lassandro G, et al. B-cell hyperfunction in children with immune thrombocytopenic purpura persists after splenectomy. Ped Res. 2016;79(2):262-270. doi:10.1038/pr.2015.211

19. Yokomichi H, Tanaka-Taya K, Koshida R, et al. Immune thrombocytopenic purpura risk by live, inactivated and simultaneous vaccinations among Japanese adults, children and infants: a matched case-control study. Int $J$ Hematol. 2020;112(1):105-114. doi:10.1007/s12185-020-02866-1

20. Vaccine adverse event reporting system. Available from: https://vaers. hhs.gov. Accessed February 15, 2021.

21. Tarawneh $\mathrm{OH}$, Tarawneh HS. Immune thrombocytopenia in a 22-year-old post Covid-19 vaccine. Am J Hematol. 2021. doi:10.1002/ajh.26106

22. Kuter DJ, Bussel JB, Lyons RM, et al. Randomized, controlled, 6-month evaluation of AMG-531 in patients with chronic immune thrombocytopenic Purpura. Lancet. 2008;371(9610):395-403. doi:10.1016/S0140-6736(08)60203-2

23. Wang B, Nichol JL, Sullivan JT. Pharmacodynamics and pharmacokinetics of AMG 531, a novel thrombopoietin receptor ligand. Clin Pharmacol Ther. 2004;76(6):628-638. doi:10.1016/j. clpt.2004.08.010

24. Papp KA, Haraoui B, Kumar D, et al. Vaccination guidelines for patients with immune-mediated disorders on immunosuppressive therapies. J of Cut Med Surg. 2019;21(1):50-74. doi:10.1177/ 1203475418811335

25. Tarantino MD, Chalmers S. Therapeutic Thrombopoietin Mimetics. In: Gresele P, Kleiman NS, Lopez JA, Page CP, editors. Platelets in Thrombotic and Nonthrombotic Disorders: Pathophysiology, Pharmacology and Therapeutics, $2^{\text {nd }}$. Cambridge University Press; 2016: 1417-1429.

26. Kuter DJ, Bussel JB, Newland A, et al. Long-term treatment with romiplostim in patients with chronic immune thrombocytopenia: safety and efficacy. Brit $J$ of Haematol. 2013;161:411-423. doi:10.1111/bjh. 12260

27. Pavord S, Thachil J, Hunt BJ, et al. Practical guidance for the management of adults with immune thrombocytopenia during the COVID-19 pandemic. Brit J of Haematol. 2020;189(6):1038-1043. doi:10.1111/bjh.16775 therapeutics; Hematology; Biotechnology/nanotechnology of blood related medicine; Legal aspects of blood medicine; Historical perspectives. The manuscript management system is completely online and includes a very quick and fair peer-review system. Visit http://www.dovepress.com/testimonials.php to read real quotes from published authors.

\section{Dovepress}

